\title{
LETTER TO THE EDITOR Breastfeeding by women with tetraplegia: some evidence for optimism
}

Spinal Cord (2014) 52, 255; doi:10.1038/sc.2013.167; published online 21 January 2014

I recently read with interest the featured case report of unilateral postpartum hypogalactia in a woman with right-sided hemiplegia due to Brown-Séquard-plus spinal cord injury. ${ }^{1}$ As pointed out by the authors, this article highlights the need for appropriate medical advice for women who may have impaired breastfeeding ability after spinal cord injury.

The case study raises the issue of difficulty in breastfeeding after cervical level spinal cord injury, but it did not mention some interesting and more positive findings regarding breastfeeding that should be relevant to clinicians advising patients with tetraplegia. As this topic is rare, it is important to point out that anecdotal reports of women with tetraplegia successfully breastfeeding exist, ${ }^{2,3}$ as does a case series detailing the difficulties and solutions used to overcome impairment to the milk ejection ('let-down') reflex in three women with absent or reduced innervation of the breasts due to tetraplegia. ${ }^{4}$

One might conclude from the case study of Liu and Krassioukov ${ }^{1}$ that women with injury rostral to $\mathrm{T} 1$ will have impaired milk production and a reduced or absent ability to breastfeed due to the absence of sympathetic innervation to the breasts. In contrast, the case studies described by Cowley ${ }^{4}$ indicate that women with tetraplegia can successfully maintain lactation and breastfeed for several months to a year postpartum, despite more extensive and bilateral impairment to both afferent and descending spinal paths than that occuring in Brown-Séquard-plus injury. ${ }^{1}$

Two of the mothers described by Cowley ${ }^{4}$ showed normal lactogenesis (including one with C5/6 ASIA A injury), and the reduced lactogenesis observed in the third mother was corrected once methods to elicit the milk-ejection reflex were implemented. Therefore impairment to the sensory-afferent component of the milkejection reflex, and subsequent reduced emptying, was thought to be primarily responsible for the hypogalactia observed in the third mother. This is consistent with the idea that milk production is localized to the breast tissue and that milk production switches from hormonal to autocrine control after stage II lactogenesis. ${ }^{5}$ The featured case report ${ }^{1}$ also noted that light touch sensation was reduced on the right breast in the woman with Brown-Sequard-plus injury indicating reduced or absent afferent input in addition to an absence of sympathetic innervation on the right. It is unclear from the article $^{1}$ if the woman with Brown-Séquard-plus syndrome used a dual breast pump to take advantage of simultaneous elicitation of the milk-ejection reflex or instead used a single pump (which would explain the hypogalactia on the right due to repeated reduced emptying secondary to impaired sensation and elicitation of milk ejection).

The observation that it is possible for women with injury rostral to T1 to breastfeed provides positive support for encouraging women with tetraplegia to attempt breastfeeding after injury, and the methods described to facilitate milk ejection when needed ${ }^{4}$ may be relevant for clinicians when advising women reporting difficulty in breastfeeding.

\section{CONFLICT OF INTEREST}

The author declares no conflict of interest.

KC Cowley

Department of Physiology, Faculty of Medicine, University of Manitoba, Winnipeg, Manitoba, Canada E-mail: kris@scrc.umanitoba.ca

1 Liu N, Krassioukov AV. Postpartum hypogalactia in a woman with Brown-Sequard-plus syndrome: a case report. Spinal Cord 2013; 51: 794-796.

2 Robertson DN. Pregnancy and labour in the paraplegic. Paraplegia 1972; 10 : 209-212.

3 Craig DI. The adaptation to pregnancy of spinal cord injured women. Rehabil Nurs 1990; 15: 6-9.

4 Cowley KC. Psychogenic and pharmacologic induction of the let-down reflex can facilitate breastfeeding by tetraplegic women: a report of 3 cases. Arch Phys Med Rehabil 2005; 86: 1261-1264.

5 Lauwers J, Shinskie D. The Science of Lactation. In: Lauwers J and Shinskie D (eds) Counseling the Nursing Mother: A Lactation Consultant's Guide, 3rd edn Jones and Bartlett: Sudbury, MA, USA, 2000, p 87-106. 